\title{
¿Cómo determinan los niños la concordancia de género?: Refutación de la teoría del género natural
}

\author{
MIGUEL PÉREZ \\ Universidad de Santiago de Compostela
}

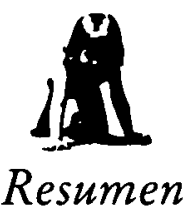

En este artículo se presentan los datos de un experimento realizado sobre el desarrollo del género con 160 niños hispanoparlantes de 4 a 11 años de edad. Las tres posibles claves (información semántica, morfofonológica, y sintáctica) que los bablantes pueden emplear para determinar el género del nombre y su concordancia con otros elementos variables que lo acompañan, han sido manipuladas experimentalmente para ofrecer las combinaciones posibles. Asi se han presentado 1) items en los que solamente estaba presente una clave, 2) items en los que babia un efecto combinado de dos de ellas en concordancia lambas ya sea masculinas, o femeninas), 3) e items en los que las claves estaban en conflicto (una de ellas era masculina y la otra femenina, por ejemplo).

Los resultados hallados (que se comparan con los obtenidos en otras lenguas) dan apoyo a la perspectiva teórica que defiende que los niños prestan más atención a la información sintáctica y morfofonológica (intralingüistica) que a la semántica (extralingüistica).

Palabras clave: Adquisición del género, Desarrollo lingüistico, Teorias del desarrollo lingüistico, Factores intralingüisticos.

\section{How do children establish gender agreement?: Refutation of the theory of natural gender}

\section{Abstract}

Data from an experiment on gender development in 160 Spanish children from 4 to 11 years of age are presented in this paper. In the Spanish language there are three possible information clues (semantic, morphophonologic, and syntactic) whick speakers can use to determine the gender of a noun and its agreement with other variable elements acompanying it. In this study, these where experimentally, manipulated to produce all possible combinations. Thus subjects were presented 1) items in which only one of the clues was present, 2) items with a combined effect of two clues in agreement (i.e., both of them feminine, or masculine), and 3) items with clues in conflict (e.g., one of them masculine and the other feminine). This experimental manipulation enabled us to test the relative strength of the different types of competing clues.

The results obtained with Spanish speaking children (which are compared with those obtained in other languages) supports the theoretical view that children pay far more attention to syntactic and morphophonological (intralinguistic) information than to semantic (extralinguistic) information.

Keywords: Gender acquisition. Language development. Intralinguistic factors. Theories of language acquisition.

Agradecimientos: Una versión diferente ha sido enviada para su publicación al Journal of Child Language. El autor desea agradecer a Dan Slobin y A. Cruttenden sus valiosos comentarios sobre una versión anterior del trabajo, y también a Guillermo Rodríguez su colaboración en la aplicación de la prueba.

Dirección del autor: Departamento de Psicología Evolutiva y de la Educación. Facultad de Filosofía y Ciencias de la Eduación. Universidad de Santiago. 15705 Santiago de Compostela.

Original recibido: Noviembre 1989. Revisión recibida: Febrero 1990. Aceptado: Marzo, 1990 


\section{INTRODUCCION}

El desarrollo del género es un tema que puede ser abordado desde diferentes puntos de vista. Uno de ellos consiste en estudiar la forma en que los niños llegan a dominar y automatizar la marcación lingüistica del género de los sustantivos, adjetivos, y otras partes del sistema lingüístico que diferencian su forma masculina y femenina, así como la concordancia entre estos elementos dentro de la oración. Los hablantes de las lenguas que establecen esa diferenciación en cuanto al género llegan a realizarse de una manera automática, sin que aparentemente les plantee dificultades. No obstante, la dificultad del proceso la podemos apreciar en los abundantes errores que cometen aquellas personas cuyo primer lenguaje no establece esta diferenciación (por ejemplo, los ingleses, o los turcos) y que tratan de aprender una segunda lengua en la que sí se marca esta diferencia (por ejemplo, el español, el francés, o el hebreo).

«Grosso modo» existen dos tipos de explicaciones sobre la forma en que los niños adquieren el género.

1) Por un lado están aquellos autores que defienden lo que se puede denominar «teoría del género natural». Según ella, el género natural de los seres animados que presentan dimorfismo sexual es lo que guía a los niños a establecer la distinción lingüística del género de los nombres correspondientes, al principio. Posteriormente esa diferenciación en cuanto al género será extendida a otros nombres, como los de los objetos, que son asexuados $\mathrm{y}$, por tanto, semánticamente arbitrarios. Evolutivamente, los niños aprenderán a marcar antes el género de los referentes animados y con rasgos semánticos. Según los defensores de esta explicación, los niños no prestan atención a elementos lingüísticos que proveen información sobre el género, como los sufijos y otros elementos sintácticos como los determinantes (artículos, demostrativos, ordinales, etc.). Son los rasgos extralingüísticos los que guían el establecimiento del género lingüístico. Mulford (1983, 1985; pero véase tambíen, aunque de una forma menos rígida, Mills, 1986) se encuentra entre los defensores máximos de esta teoría, que se puede considerar como una derivación de una teoría más general de la adquisición del lenguaje. Según ella los niños, una vez que han adquirido una nueva noción, deben aprender su correspondiente forma de expresión lingüística, o, dicho de otra forma, el aprendizaje del lenguaje consiste en el establecimiento de relaciones entre formas y significados. El nombre de «teoria de base semántica» con que es conocida refleja bastante bien la idea de que lo primero es lo conceptual (véase, por ejemplo, MacNamara, 1972; Slobin, 1973; Schlesinger, 1981).

2) Opuesta a la anterior es la teoría que sostiene que para la adquisición y desarrollo del género lingüístico los niños se apoyan fundamentalmente en la información que proporciona el propio lenguaje y el contexto lingüístico en que aparecen las palabras, siendo la información morfológica y sintáctica la más importante (Karmiloff-Smith, 1979; Levy, 1983, 1988). Los niños son capaces de captar claves informacionales que proporcionan las terminaciones de las palabras o de apreciar de qué otras palabras van acompañadas para establecer su género. Análogamente se podría considerar que esta teoría es una concreción de aquélla más general que sostiene 
que los niños en la tarea de aprender a hablar también prestan atención a las propiedades formales del lenguaje, y son capaces de captar regularidades en los contextos lingüisticos en que aparecen las palabras (Maratsos, 1983, 1988; Maratsos y Chalkely, 1980; Karmiloff-Smith, 1983, 1986). La información que proporciona el propio sistema lingüístico, o información intralingüistica, se convierte en objeto de la atención intelectual del niño. Esta visión es coincidente con una idea ya expresada por Bates, Benigni, Bretherton, Camaioni y Volterra (1977) según la cual no existe un área privilegiada de experiencia en la cual el niño forje su desarrollo, tal como se deriva de la teoría piagetiana, en la cual se prima el papel de la experiencia física. Tanto el ámbito de la experiencia física, como el de la social, o la lingüística, exigirán el trabajo del niño, y suscitarán su interés. Tal como ha expresado Karmiloff-Smith (1983) el lenguaje constituye un espacio formal que exige la solución de problemas específicos.

La investigación que aquí se presentará trata de establecer la importancia que para los niños tienen los rasgos intralingüísticos y extralingüísticos en el establecimiento de la concordancia del género y en la determinación del género de los sustantivos.

Por investigaciones anteriores se sabe que los niños son capaces de captar el dimorfismo sexual desde la edad de 30 meses aproximadamente. Es entonces cuando pueden identificar una figura como de un hombre o de una mujer, y clasificarse a si mismos como perteneciendo a uno de estos tipos. Se dice que es en ese momento cuando los niños han adquirido la identidad de género (Fagot, Leinbach, y Hagan, 1986; López, 1988). La relevancia del género como una dimensión para la clasificación -lo que se ha denominado el empleo del esquema de género (Bem, 1981) - se hace mayor justo antes de que la permanencia del género, o idea de que el rol sexual de cada uno es algo permanente con independencia de cambios externos de apariencia, sea adquirida, cosa que ocurre alrededor de los 6 u 8 años de edad (Serbin y Sprefkin, 1986; Gelman, Collman, y Maccoby, 1986; López, 1984, 1988). Por tanto, estas investigaciones nos han permitido saber que los niños, efectivamente, le prestan atención a las claves genéricas extralingüísticas desde una edad muy temprana.

Pero otras investigaciones también han puesto de relieve que los niños son capaces de procesar claves intralingüísticas (cf. Pérez Pereira, 1988). Por ejemplo, los niños hacia los 2 años de edad son capaces de distinguir entre nombres contables e incontables (Gordon, 1985; Gathercole, 1985) o propios y comunes (Katz, Baker, y MacNamara, 1974) atendiendo a los patrones distribucionales o contextos lingüísticos en que aparecen. Mas directamente relacionado con esta investigación, Tucker, Lambert, y Rigault (1977) han demostrado la capacidad de los niños francoparlantes para decidir cuál es el género de nombres artificiales en base a su terminación morfofonológica.

Aun cuando investigaciones como las reseñadas han establecido que los niños pueden utilizar la información fonológica, sintáctica, y extralingüística, sin embargo los estudios realizados sobre la adquisición del género no han explorado nunca sistemáticamente cuáles son las claves informacionales que utilizan los niños - y a qué edades - para decidir el género de las palabras y para establecer la concordancia de género, o, mejor aún, cuál es la importancia relativa de cada una de ellas. Indudablemente, ésta sería la 
forma más adecuada de someter a prueba las dos posiciones teóricas mencionadas antes.

En lo que concierne a los estudios realizados sobre el tema específico de la adquisición y el desarrollo del género lingüístico, se pueden apreciar dos tipos diferentes. 1) El primero lo.forman aquellas investigaciones longitudinales o transversales que han recogido datos del habla de los niños en contextos naturales (Popova, 1973; Henzel, 1975; McWhinney, 1978; Berman, 1981, 1985; Levy, 1983a, 1983b; Hernández Pina, 1984; Mills, 1986). Su interés primordial reside, además de en la secuencia de adquisiciones que establecen, en el análisis de los errores producidos por los niños, y en la comparación translingüística de los procesos evolutivos que posibilitan. Sin embargo, este tipo de estudios, a pesar de su gran utilidad, no nos permite apreciar la importancia relativa de las claves intralingüísticas y extralingüísticas en el aprendizaje del sistema lingüístico del género, de la forma que podrían hacerlo estudios experimentales. 2) El segundo tipo está integrado por aquellos estudios experimentales en que se trata de probar la importancia de las claves intra o extralingüísticas en la adquisición del sistema del género, o una parte de él, por parte de los niños. Han sido realizados con niños que hablan diferentes lenguas, tales como el alemán, el francés, el islandés, o el inglés (Deutsch y Pechman, 1978; Karmiloff-Smith, 1979; Mulford, 1985; Mills, 1986). Desafortunadamente, no siempre han sido lo necesariamente rigurosos metodológicamente como para poder extraer de ellos resultados firmes (cf. Levy, 1988; Pérez Pereira, no publicado).

El estudio de la adquisición del género, además de su pertinencia para comprobar la adecuación o no de las teorías del género natural y formal, respectivamente, presenta también una serie de implicaciones sobre la forma de concebir las relaciones entre lenguaje y pensamiento. Muy sucintamente, se puede decir que si se defiende la primera de ellas, se apoya la hipótesis del determinismo cognitivo (cf. Pérez Pereira, 1984), también denominada hipótesis cognitiva fuerte (García Madruga, 1988). Según ésta, se considera que el desarrollo del pensamiento es previo a y dirige el desarrollo lingüístico. En definitiva, la forma en que se produce el desarrollo del lenguaje depende del desarrollo cognitivo, de la forma en que los niños se representan la realidad en distintas edades, el tipo de nociones que poseen, etc. La vinculación de esta hipótesis con la teoría del aprendizaje lingüístico de base semántica es evidente. Por el contrario, si se defiende la segunda estará mucho más de acuerdo con una teoría que admita una relativa autonomía del desarrollo lingüístico respecto del desarrollo cognitivo, aún cuando se hable de interrelaciones entre ellos en todas las direcciones, de tal manera que también el ámbito de la experiencia lingüística influya sobre el desarrollo cognitivo del sujeto (véase Pérez Pereira, 1984, para una exposición detallada de esto).

Para ser concretos, el objetivo del presente estudio es el de determinar la cuestión de la relativa importancia de las claves de información intra y extralingüística en lo que se refiere a la habilidad de los niños españoles para reconocer el género de un nombre escuchado en un determinado marco, y, consecuentemente, establecer la concordancia de otros elementos variables que lo acompañan. En esta investigación se ha realizado un esfuerzo por superar defectos metodológicos que aparecen en otros estudios experimentales. 
En español existe una tendencia a que los nombres acabados en -o sean masculinos, mientras que los acabados en -a tienden a ser femeninos. Esta tendencia a que las terminaciones en -o sean masculinas y las terminaciones en -a sean propias del femenino (Rosemblat, 1962; Anderson, 1961), se extiende también a los plurales (terminaciones en -os/-as), y a los diminutivos. Además de los sustantivos, otras partes del habla que concuerdan con aquellos presentan también alternancia de género. Entre éstas se hallan los determinantes que preceden al nombre, y los adjetivos calificativos que normalmente lo siguen (cf. Seco, 1973, Martínez, 1977).

Por tanto, tenemos que en español hay tres marcas que nos permiten decidir cuál es el género de un nombre y su concordancia con otras palabras de la oración, como los adjetivos calificativos (cf. Ibrahim, 1973). Estas son, la información extralingüistica, que proviene de los rasgos sexuales del referente, la información morfofonológica, que nos proporciona el sufijo de la palabra, y la información sintáctica, aportada por el género del determinante (artículo, adjetivo demostrativo...), por ejemplo. Sin embargo no todas están siempre presentes. Algunas veces el sufijo de un nombre no nos proporciona ninguna indicación acerca de su género, o el determinante puede ser un numeral sin marca de género, o el referente puede ser una cosa. En la Tabla I se presentan algunos ejemplos de estas posibilidades.

\section{TABLA I}

Ejemplos de claves del nombre y del determinante que pueden ser utilizadas para establecer la concordancia de género de los adjetivos*

1) Clave morfofonológica: terminación del nombre en -o/ -a:

Dos libros rojos

Dos pañoletas rojas

2) Clave sintáctica: artículo precedente masculino o femenino:

El patín blanco

La nieve blanca

3) Género natural: rasgos sexuales:

Dos hombres honestos

Dos mujeres honestas

4) Ninguna clave:

Dos árboles altos

Dos naves largas

5) Todas las claves están presentes:

Un italiano sincero

Una americana guapa

* El marcador lingüistico está subrayado. El orden de los elementos es siempre Determinante-Nombre-Adjecivo.

El español, al no mezclar la variación de género con la variación de caso (como le ocurre, por ejemplo al alemán, ruso, o servo-croata), permite variar a voluntad los elementos de marcación del género sin producir efectos secundarios. De esta forma constituye una lengua muy apropiada para poner a prueba la importancia relativa de las tres claves indicadas. 


\section{METODO}

Teniendo en cuenta que hay tres claves que los niños pueden utilizar para reconocer el género de una palabra desconocida al oírla en un contexto lingüístico determinado, y para, consiguientemente, establecer la concordancia de otros elementos variables que la acompañan, se ha realizado un diseño experimental que incluye todas las combinaciones posibles entre esas claves. Con ello se pretendía que fuera posible comparar los efectos de cada una de ellas, dado que se esperaba que las respuestas de los niños mostrarían cuál era el género que ellos atribuían a nombres artificiales.

La Tabla II contiene las claves presentadas en cada item. En total había 22 items. Se presentaron diferentes tipos de items: 1) items en los que solamente estaba presente una clave (items 1 al 6 en la Tabla II);2) items en los que había un efecto combinado de dos de ellos en concordancia (items 7 a 12 de dicha Tabla); 3) items en los que las claves estaban en conflicto (una de ellas era masculina, mientras que la otra era femenina, por ejemplo, o dos de ellas femeninas y la otra masculina; items 12 a 22 de la Tabla II). Esta manipulación experimental permitió comprobar la fuerza relativa de las diferentes claves en competencia (véase Gordon, 1985; y Kail y Charvillat, 1988 para una justificación del procedimiento).

Aquellas situaciones en que estaban presentes las tres claves en concordancia, o todas ellas ausentes, fueron deliberadamente excluidas, puesto que no aportaban ningún conocimiento útil.

Se podría considerar, por tanto que éste es un diseño factorial de $3 \times 3$ ( $\sin$ tener en cuenta la variación en la edad de los sujetos ${ }^{8}$ ).

TABLA II

Claves ofrecidas en cada item *

\begin{tabular}{rccll}
\hline & Determinante & Género natural & Sufijo & \multicolumn{1}{c}{ Ejemplo } \\
\hline 1 & $\mathrm{M}$ & $\mathrm{O}$ & $\mathrm{O}$ & un tantén \\
2 & $\mathrm{~F}$ & $\mathrm{O}$ & $\mathrm{O}$ & una pilín \\
3 & $\mathrm{O}$ & $\mathrm{M}$ & $\mathrm{O}$ & dos palires \\
4 & $\mathrm{O}$ & $\mathrm{O}$ & $\mathrm{O}$ & dos borales \\
5 & $\mathrm{O}$ & $\mathrm{O}$ & $\mathrm{M}$ & dos carepos \\
6 & $\mathrm{O}$ & $\mathrm{M}$ & $\mathrm{O}$ & dos aniras \\
7 & $\mathrm{M}$ & $\mathrm{F}$ & $\mathrm{O}$ & una talaz \\
8 & $\mathrm{~F}$ & $\mathrm{O}$ & $\mathrm{F}$ & una lodena \\
9 & $\mathrm{~F}$ & $\mathrm{M}$ & $\mathrm{M}$ & un nepo \\
10 & $\mathrm{M}$ & $\mathrm{F}$ & dos milipos \\
11 & $\mathrm{O}$ & $\mathrm{F}$ & $\mathrm{O}$ & dos ticas \\
12 & $\mathrm{O}$ & $\mathrm{M}$ & $\mathrm{O}$ & una liben \\
13 & $\mathrm{M}$ & $\mathrm{O}$ & $\mathrm{M}$ & una linolo \\
14 & $\mathrm{~F}$ & $\mathrm{O}$ & $\mathrm{F}$ & un satila \\
15 & $\mathrm{~F}$ & $\mathrm{~F}$ & $\mathrm{M}$ & dos lacatos \\
16 & $\mathrm{M}$ & $\mathrm{M}$ & $\mathrm{F}$ & dos danitas \\
17 & $\mathrm{O}$ & $\mathrm{F}$ & $\mathrm{M}$ & una rebo \\
18 & $\mathrm{O}$ & $\mathrm{M}$ & $\mathrm{F}$ & una tiba \\
19 & $\mathrm{~F}$ & $\mathrm{~F}$ & $\mathrm{~F}$ & un capola \\
20 & $\mathrm{M}$ & $\mathrm{M}$ & $\mathrm{M}$ & una lampo \\
21 & $\mathrm{M}$ & $\mathrm{F}$ & & \\
22 & & & & \\
\hline
\end{tabular}

* El género de cada clave está indicado por M (masculino), $\mathrm{F}$ (femenino), u O (ausente). 


\section{Material}

Se han utilizado dibujos coloreados de seres, animales, o cosas, imaginarios. Algunos de ellos representaban seres que poseían rasgos sexuales secundarios, mientras que otros no presentaban género natural. Cada uno de estos dibujos tenía asociado un nombre artificial, y, por tanto, desconocido para los niños. Estos nombres inventados podían ser considerados como masculinos, femeninos, o indefinidos, en función de sus terminaciones morfofonológicas. La Figura I presenta algunos ejemplos de los dibujos utilizados, y la tabla III muestra la lista de palabras artificiales.
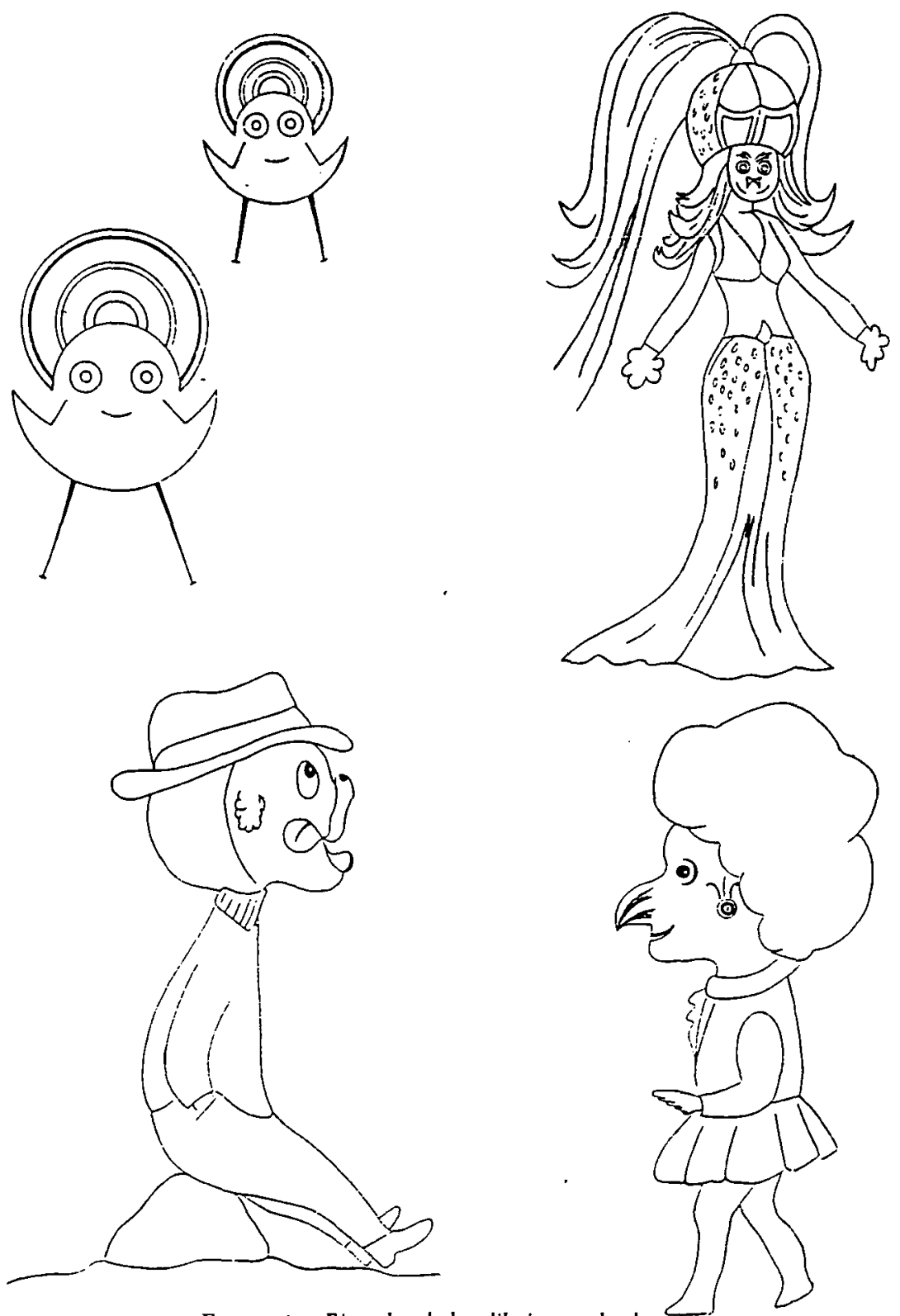

FIGURA 1. Ejemplos de los dibujos empleados. 
TABLA III

Lista con las palabras artificiales empleadas

\begin{tabular}{lll}
\hline \multicolumn{1}{c}{ Género masculino } & \multicolumn{1}{c}{ Género femenino } & Género no marcado \\
\hline linolo & satila & pifar \\
lampo & capola & liben \\
milipos & ticas & palires \\
nepo & lodena & borales \\
carepo & aniras & cater \\
rebo & tiba & talaz \\
lacatos & danitas & tantén \\
& & pilin \\
\hline
\end{tabular}

Las instrucciones verbales utilizadas por el experimentador podían llevar un artículo femenino (la, las, una, unas), un artículo masculino (el, los, un, unos), o una palabra que no aportase ninguna información acerca del género del nombre (el numeral dos) antes de él.

\section{Sujetos}

El experimento ha sido aplicado a 160 sujetos hispanoparlantes distribuidos en 8 grupos de edad con 20 sujetos cada uno desde 4 a 11 años, $y$ cuyas edades medias eran $4 ; 4,5 ; 4,6 ; 7,7 ; 6,8 ; 5,9 ; 4,10 ; 6$, y $11 ; 6$. El número de niños y de niñas era el mismo para cada grupo. Todos ellos procedían de un entorno sociocultural bajo o medio-bajo de las ciudades de Santiago de Compostela y Vigo.

\section{Procedimiento}

El procedimiento utilizado está inspirado en el que utilizó KarmiloffSmith (1979). Los dibujos se presentaban individualmente a cada niño, mediante la siguiente instrucción tipo: (1) «Aquí hay un pifar», o «este es el dibujo de un pifar». A continuación se le mostraba un dibujo exactamente igual, pero de diferente color, y se le preguntaba al niño; (2) "Aquí hay otro dibujo, ¿qué piensas que es?» o "¿qué hay allí? Si el niño no especificaba el color del dibujo se le preguntaba «¿de qué color?» Después se realizaba cualquier acción con la tarjeta (se ocultaba, se giraba $180^{\circ}$, o se colocaba algún objeto encima), y se le preguntaba al niño: (3) « ¿Qué he hecho?» Cuando la respuesta del niño no era suficientemente clara, se utilizaban expresiones que no aportaban ninguna información adicional acerca del género de los hombres, tal como «¿cuál?», «¿qué pifar?", "¿qué es?»

Sobre este formato de preguntas se insertaba adecuadamente la información verbal y no verbal que proveía cada item.

Los adjetivos producidos por los niños constituían el criterio para determinar la atribución del género del nombre y de la concordancia de género que realizaban. Para posibilitar esto, los colores de los dibujos utilizados presentaban una clara diferenciación morfofonológica en cuanto al género en el sistema lingüístico: rojo/roja, blanco/blanca, negro/negra, y amarillo/amarilla. De tal manera que, por ejemplo,.si un niño respondía al item 6 de la Tabla IV "Ahora has tapado los borales rojos", era evidente 


\section{1}

TABLA IV

Orden de presentación de los items con una análisis de las claves ofrecidas en cada uno de ellos

\begin{tabular}{|c|c|c|c|c|}
\hline & Items & Artículo & Género natural & Sufijo \\
\hline & un pifar & M & $\mathrm{F}$ & $\mathrm{O}$ \\
\hline 2 & una liben & $\mathrm{F}$ & $M$ & O \\
\hline 3 & una linolo & $\mathrm{F}$ & 0 & $M$ \\
\hline 4 & un satila & M & $\mathrm{O}$ & $\mathrm{F}$ \\
\hline & dos palires & $\mathrm{O}$ & $\mathrm{M}$ & $\mathrm{O}$ \\
\hline & dos borales & $\mathrm{O}$ & $\mathrm{F}$ & $\mathrm{O}$ \\
\hline 7 & un capola & $\mathrm{M}$ & $\mathrm{F}$ & $\mathrm{F}$ \\
\hline & una lampo & $\mathrm{F}$ & $\mathrm{M}$ & M \\
\hline 9 & dos milipos & $\mathrm{O}$ & M & M \\
\hline 10 & dos ticas & $\mathrm{O}$ & $\mathrm{F}$ & F \\
\hline 11 & una lodena & $\mathrm{F}$ & $\mathrm{O}$ & $\mathrm{F}$ \\
\hline 12 & un nepo & $\mathrm{M}$ & 0 & M \\
\hline 13 & un cater & $\mathrm{M}$ & M & $\mathrm{O}$ \\
\hline 14 & una talaz & $\mathrm{F}$ & $\mathrm{F}$ & 0 \\
\hline 15 & dos carepos & $\mathrm{O}$ & 0 & M \\
\hline 16 & dos aniras & $\mathrm{O}$ & $\mathrm{O}$ & $\mathrm{F}$ \\
\hline 17 & una rebo & $\mathrm{F}$ & $\mathrm{F}$ & M \\
\hline 18 & un tiba & M & M & $\mathrm{F}$ \\
\hline 19 & un tantén & M & $\mathrm{O}$ & $\mathrm{O}$ \\
\hline 20 & una pilin & $\mathrm{F}$ & $\mathrm{O}$ & $\mathrm{O}$ \\
\hline 21 & dos lacatos & $\mathrm{O}$ & $\mathrm{F}$ & M \\
\hline & dos danitas & $\mathrm{O}$ & M & $\mathbf{F}$ \\
\hline
\end{tabular}

que estaba considerando el nombre de dicho item como masculino, y estaba estableciendo la concordancia entre el adjetivo y el nombre de acuerdo con esa suposición.

El posible efecto de orden de presentación de los items fue controlado mediante la utilización de un orden inverso para la mitad de los sujetos de cada grupo de edad. Para cada par, los items masculinos y femeninos fueron contrabalanceados para evitar el efecto de orden que se daria si el primer item de cada par fuera siempre masculino, por ejemplo. La Tabla IV contiene el orden de presentación de los items para la mitad de los sujetos. Para la otra mitad el orden fue invertido, tal como ya se ha indicado.

\section{RESULTADOS}

La Tabla $\mathrm{V}$ contiene los resultados generales dados en porcentajes.

Aplicando el test de $\mathrm{X}^{2}$, no se ha hallado ninguna diferencia en los resultados debida al orden de aplicación de los items. Tampoco se ha hallado diferencia entre niños y niñas.

Al comparar los grupos de edad, se ha hallado una diferencia global entre ellos en el item 18 (OMF) de la Tabla I $\left(\mathrm{X}^{2}=14,24, \mathrm{p}<0,05\right)$, y diferencias parciales entre los grupos de edad de 7 y 9, y 8 y 9 años $\left(X^{2}=6,4\right.$, $p<0,025)$, entre los grupos de 7 y 6, y 8 y 6 años $\left(X^{2}=4,6, p<0,05\right)$, y entre los grupos de 9 y 11 , y 9 y 4 años $\left(X^{2}=4,88, p<0,05\right)$.

En el item 21 (MFF) se han encontrado diferencias significativas entre los grupos de 6 y 9 años, y entre los grupos de 6 y 10 años de edad $\left(\mathrm{X}^{2}=3,94, \mathrm{p}<0,05\right)$. Ninguna otra diferencia significativa ha sido hallada 
TABLA V

Resultados generales en porcentajes

\begin{tabular}{|c|c|c|c|c|c|c|c|c|c|}
\hline \multirow{2}{*}{ Item } & \multirow{2}{*}{$\begin{array}{l}\text { Género elegido } \\
\text { por el niño }\end{array}$} & \multicolumn{8}{|c|}{ Grupo de edad } \\
\hline & & 4 & 5 & 6 & 7 & 8 & 9 & 10 & 11 \\
\hline $1 \mathrm{MOO}$ un tantén & Género & 95 & 95 & 100 & 100 & 100 & 95 & 100 & 100 \\
\hline $2 \mathrm{~F}$ & Gé & 65 & 75 & 85 & 85 & 65 & 80 & 90 & \\
\hline 3 OMO dos $p$ & Gé & 100 & 100 & 1001 & 1001 & 100 & 95 & 100 & 100 \\
\hline 4 OFO dos bc & Género & & 15 & 10 & 5 & 0 & 20 & 5 & 20 \\
\hline 5 OOM d & $\mathrm{G}$ & 100 & 100 & 1001 & 1001 & 100 & 100 & 100 & 100 \\
\hline 6 OOF & $\mathrm{G}$ & 95 & 80 & 70 & 65 & 85 & 80 & 70 & \\
\hline $7 \mathrm{MMO}$ & & 100 & 100 & 951 & 1001 & 100 & 100 & 100 & 100 \\
\hline $8 \mathrm{FF}$ & $G$ & 80 & 80 & 95 & 90 & 90 & 90 & 90 & 85 \\
\hline 9 FOF & $\mathrm{G}$ & 95 & 90 & 1001 & 1001 & 100 & 100 & 100 & 100 \\
\hline $10 \mathrm{MON}$ & G & 100 & 100 & 1001 & 1001 & 100 & 100 & 100 & 100 \\
\hline $11 \mathrm{OMM}$ & Géner & 100 & 100 & $100 i$ & 1001 & 100 & 100 & 100 & $\begin{array}{r}100 \\
90\end{array}$ \\
\hline 12 OFF & Género & $\begin{array}{l}90 \\
95\end{array}$ & $\begin{array}{l}95 \\
95\end{array}$ & $\begin{array}{r}100 \\
95\end{array}$ & 95 & $\begin{array}{r}95 \\
100\end{array}$ & 90 & $\begin{array}{r}100 \\
90\end{array}$ & 90 \\
\hline $14 \mathrm{FMO}$ & Género femenino & 90 & 75 & 85 & 70 & 75 & $\begin{array}{l}73 \\
70\end{array}$ & 90 & 75 \\
\hline $15 \mathrm{~F}$ & Género femenino & 40 & 55 & 45 & 55 & 30 & 55 & 60 & 60 \\
\hline $16 \mathrm{M}$ & Géne & 85 & 75 & 85 & 75 & 90 & 85 & 85 & \\
\hline 17 OFM dos lacatos & Género masculino & 85 & 100 & 95 & 95 & 95 & 80 & 85 & 95 \\
\hline 18 OMF dos danitas & Género femen & 65 & 55 & 35 & 70 & 70 & 30 & 45 & \\
\hline $19 \mathrm{FFM} \mathrm{u}$ & Género femenino & 65 & 85 & 90 & 75 & 75 & 100 & 95 & 85 \\
\hline $20 \mathrm{MMF}$ un tiba & Género masculino & 80 & 100 & 90 & 951 & 100 & 90 & 85 & \\
\hline $21 \mathrm{MFF}$ & Género fem & 25 & 40 & 50 & 45 & 25 & 20 & 20 & 30 \\
\hline 22 FMM una lampo & Género masculino & 55 & 50 & 35 & 45 & 40 & 45 & 25 & 30 \\
\hline
\end{tabular}

- A lo largo de todo el trabajo se indica el género de cada clave mediante M (masculino), F (femenino), u O (ausente), colocados en una posición fija, de tal manera que la primera posición está ocupada por la clave sintáctica (determinante), la segunda posición por la clave extralingüistica (género natural), y la tercera por la clave morfológica (sufijo de la palabra). Por ejemplo (OMF) indica que el determinante es neutro (numeral «dos*), el género natural es masculino (un macho), y el sufijo del nombre es femenino (terminación en $/-\mathrm{a} /$ ).

entre los grupos de edad en los restantes items, pero es digno de ser señalado que en el item 22 (FMM) la diferencia obtenida casi es significativa entre los resultados de los grupos de edad de 4 y 10 años $\left(\mathrm{X}^{2}=3,74<3,84\right.$ para $a=0,05)$ y entre los de 4 y 11 años $\left(X^{2}=3,62\right)$.

Estos resultados indican que apenas existe cambio evolutivo entre los 4 y los 11 años de edad. Los resultados del item 22 y 21 indican que los niños más mayores prestan más atención a la información sintáctica que a la información morfológica en aquellos items en que hay un conflicto entre claves, puesto que los porcentajes de respuestas femeninas son mayores en el item 22 que en el 21.

La falta de concordancia entre el género del adjetivo y el género del artículo, como en atapaste la linolo blanco» se ha producido en solamente un $3,6 \%$ de las respuestas, y mucho más frecuentemente en niños de 4 y 5 años. Esta falta de concordancia se dio especialmente en los items 15 (FMO), 22 (FMM), 19 (FFM), 2 (FOO), 14 (FMO), y 8 (FFO) (frecuencias de $24,17,14,14,13$, y 11 respectivamente). Este resultado parece indicar que incluso los sujetos más jóvenes han adquirido ya la concordancia de género, pues solamente los items con contradicción entre claves presentan algún problema a los niños a causa de su ambivalencia, como, por otra parte, sería lógico esperar.

La comparación de las diferencias entre items fue comprobada también mediante la aplicación de la prueba de $\mathrm{X}^{2}$ para datos relacionados. Los aná- 
lisis estadísticos fueron realizados evitando aquellos items con claves masculinas únicamente, dado que los altos resultados observados han producido un «efecto de techo". Los resultados más interesantes son los siguientes:

1) Diferencia significativa entre los items 1 y 2 (MOO $>$ FOO) $\left(\mathrm{X}^{2}=25, \mathrm{p}<0,001\right), 3$ y 4 (OMO $>$ OFO) $\left(\mathrm{X}^{2}=146, \mathrm{p}<0,001\right), 5$ y 6 (OOM $>\mathrm{OOF}),\left(\mathrm{X}^{2}=34, \mathrm{p}<0,001\right)$. Estos resultados indicarían que los items con claves masculinas son más fácilmente reconocidos que los items con claves femeninas. Los niños muestran una tendencia a atribuir el género masculino a los nombres desconocidos.

2) Diferencias significativas entre los items 2 y $4(\mathrm{X}+2=114,13$, $\mathrm{p}<0,001)$ (FOO $>$ OFO), y entre los items 6 y $4\left(\mathrm{X}^{2}=103, \mathrm{p}<0,001\right)$ (OOF $>$ OFO). Lo cual revela que los items que proporcionan claves sintácticas o morfofonológicas son más claros que aquellos que poseen claves extralingüísticas. Por el contrario, no se ha hallado diferencia significativa alguna entre los items 2 y 6 (FOO = OOF), lo que indica la importancia semejante que existe entre las claves sintácticas y morfofonológicas.

3) El efecto combinado de claves en corcondancia mejora los resultados, en contraste con aquellos items en que solamente se aporta una sola clave. Esto se comprueba al comparar las respuestas femeninas en los items 9 y $2\left(\mathrm{X}^{2}=16,9, \mathrm{p}<0,001\right)(\mathrm{FOF}>\mathrm{FOO}), 9$ y $6\left(\mathrm{X}^{2}=29,12, \mathrm{p}<0,001\right)$ (FOF $>$ OOF), 12 y $6\left(\mathrm{X}^{2}=16,8, \mathrm{p}<0,001\right)(\mathrm{OFF}>\mathrm{OOF})$. No se ha encontrado diferencia significativa entre los items 8 y $2\left(\mathrm{X}^{2}=3,7<3,84\right.$ para $\mathrm{a}=0,05)(\mathrm{FFO}=\mathrm{FOO})$, lo que, una vez más, indica la escasa atención prestada a las claves extralingüísticas.

4) Se ha hallado diferencia significativa entre los resultados de los items 9 y $8\left(\mathrm{X}^{2}=17, \mathrm{p}<0,001\right)$ (FOF $>\mathrm{FFO}$ ), pero no entre los items 9 y 12 $\left(\mathrm{X}^{2}=3\right.$ ) (FOF $=$ OFF), lo que indica la mayor importancia de las claves morfofonológicas comparadas con las claves sintácticas en situaciones de acuerdo entre claves. Si las claves morfofonológica y sintáctica tuviesen un peso equivalente, deberíamos aguardar resultados similares en todos los items en los que cada una de ellas estuviese asociada con la clave de género natural en relación a los items en que ambas van juntas. Sin embargo esto no ocurre. La misma conclusión se deriva de la comparación entre los items 8 y $12\left(\mathrm{X}^{2}=4,84, \mathrm{p}<0,05\right)$. Para decirlo con otras palabras, los niños reconocen que dos ticas (OFF) es una palabra femenina con mayor facilidad que una talaz (FFO), siendo el referente una Figura femenina en ambos casos.

5) Los análisis realizados sobre los items en los que existe contradicción entre claves indican que, en esas situaciones, los niños prestan más atención a las claves sintácticas que a las morfofonológicas. Se han encontrado diferencias significativas al comparar las tasas de respuestas femeninas en los items 14 y $18\left(\mathrm{X}^{2}=18,3, \mathrm{p}<0,001\right)$ (FMO $\left.>\mathrm{OMF}\right), 15$ y 16 $\left(\mathrm{X}^{2}=39,40, \mathrm{p}<0,001\right)(\mathrm{FOM}>\mathrm{MOF}), 19$ y $21\left(\mathrm{X}^{2}=71,02, \mathrm{p}<0,001\right)$ (FFM $>$ MFF), y entre los items 20 y $22\left(\mathrm{X}^{2}=70,5, \mathrm{p}<0,001\right)$ (FMM > MFF). En todos estos casos se dan comparativamente más respuestas femeninas a los items en que el artículo es femenino que a aquellos en los que lo es el sufijo cuando la clave antagonista es masculina.

6) No se han apreciado diferencias significativas entre los items 1 y 7 $(\mathrm{MOO}=\mathrm{MMO}), 2$ y $8(\mathrm{FOO}=\mathrm{FFO})$, y 5 y $11(\mathrm{OOM}=\mathrm{OMM}$-aun 
cuando sí se han encontrado entre los items 12 y 6 (OFF > FFO)-. Esto indica que no hay una diferencia importante en la forma en que los niños manejan los nombres animados e inanimados, puesto que no responden significativamente mejor a los items con figuras animadas ( $y$, por tanto, con rasgos sexuales diferenciados) que a los que presentan figuras inanimadas. Por consiguiente, la presencia de figuras con género natural no mejora en gran medida los resultados de los niños.

7) A pesar de que las claves de género natural (sexo del referente) son menos importantes que las intralingüísticas, sí que influyen en las decisiones que toman los niños acerca del género de nombres desconocidos y su concordancia gramatical. Esto se revela especialmente en los items en que existe un desacuerdo entre el artículo y el sufijo. Tenemos dos ejemplos de ello en que a) los niños dan más respuestas femeninas en el item 19 que en el item 15 (FFM $>$ FOM) $\left(X^{2}=42,8, p<0,001\right)$, y b) también proporcionan más respuestas masculinas en el item 20 que en el 16 (MMF $>$ MOF) $\left(\mathrm{X}^{2}=7, \mathrm{p}<0,01\right)$. En ambos casos la concordancia del género natural con otra clave aumenta la probabilidad de que su género sea dado como respuesta frente al de la clave antagonista.

\section{DISCUSION}

La mayor tendencia a atribuir el género masculino a los nombres, y los altos resultados obtenidos con los items que proporcionan claves masculinas, parecen apoyar la teoría de Greemberg (1966) sobre la marcación del género. Según ella, el masculino es el término no marcado y, por tanto, más fácil de adquirir. La tendencia observada en nuestros sujetos es aún mayor que la hallada en niños franceses por Karmiloff-Smith (1979), en el uso de pronombres por niños ingleses y alemanes (Mills, 1986), y en la comprensión de pronombres por niños alemanes (Deutsch y Pechman, 1978). En los estudios longitudinales existentes, se aprecian resultados no coincidentes. Mientras algunos de ellos indican una tendencia inicial hacia el uso de formas con género masculino (por ejemplo Levy, 1983a y 1983b, y Berman, 1985, con niños hebreos), otros indican una cierta sobregeneralización de formas femeninas (por ejemplo, Mills, 1986, con niños alemanes, o Hernández Pina, 1984, con un niño español).

Nuestros resultados también indican que los niños son capaces de procesar y tener en cuenta información proveniente de diferentes claves, puesto que cuantas más claves convergentes haya, más fácilmente asignan el género a los nombres.

Un resultado primordial de esta investigación es que los niños prestan mucha más atención a las claves sintácticas y morfofonológicas que a las extralingüísticas (género natural). En lo que respecta al rasgo animado/inanimado, éste no parece jugar un papel importante en el manejo infantil de los nombres. Los resultados de esta investigación se oponen a la teoría del género natural (Mulford, 1985; pero también Mills, 1986), según la cual los niños prestan mucha más atención a los rasgos del referente - especialmente al rasgo animado/inanimado- al asignar inicialmente el género a los nombres.

A medida que los niños crecen, parece que prestan mayor atención a 
las claves sintácticas, que llegan a ser las más importantes. Este descubrimiento, que se refiere especialmente a las situaciones con claves ambiguas o contradictorias, no constituye un argumento a favor de la afirmación que hace Slobin (1985: 1218) de que los niños utilizan la fonología como una clave más fiable para el género que la forma del artículo, y consiguientemente no apoya su propuesta acerca de la jerarquía de uso de opciones en el Principio Operativo: Paradigmas Morfológicos. Aunque también podría ser que este Principio Operativo sea utilizado en un estadio inicial. Cuando el niño descubre posteriormente que la sintaxis puede invalidar la fonología, comienza a emplear las claves sintácticas, como ocurre en aquellos nombres que tienen un género aparentemente diferente, por su terminación, de su género gramatical: por ejemplo, el programa. En relación a esto hay que tener presente que nuestros sujetos más jóvenes tenían ya 4 años, y que la propuesta de Slobin se refiere a un período anterior.

En cualquier caso, es la información intralingüística, y no la extralingüística como proclama Mulford (1985), la que los niños utilizan principalmente para establecer el género de los nombres y la concordancia de otras partes del habla como el sustantivo. Mulford ha realizado un estudio experimental sobre la comprensión de pronombres en niños islandeses (Mulford, 1985), que es considerado como una de las evidencias más importantes de la teoría del género natural o teoría semántica. Sin embargo su experimento presenta serias deficiencias metodológicas que hacen de él un débil soporte de la mencionada teoría. Un análisis crítico de su experimento y resultados (ver Pérez-Pereira, no publicado) indica que la información semántica no es más importante que la información que proporcionan elementos formales (sufijos) en el caso de los niños islandeses, siendo el peso de la información semántica semejante al hallado en otros estudios experimentales (Karmiloff-Smith, 1979, y esta investigación). Una muestra de ello la tenemos en que los niños islandeses reconocen el referente de un pronombre personal con solamente algo más de facilidad en los items que contienen información semántica y morfofonológica a la vez que en los que solamente contienen información morfofonológica (sufijo) ( $75,4 \%$ frente al $65,5 \%$ de respuestas correctas, respectivamente). Por tanto, y dejando de lado las diferencias que existen entre el sistema de género en español e islandés (por ejemplo, las terminaciones usadas para marcar el género de los nombres presentan más funciones y son, por tanto, menos claras), las inconsistencias metodológicas mencionadas en el estudio de Mulford deberían prevenirnos de aceptar sus conclusiones.

Estudios realizados con niños españoles indican que estos ya han adquirido el manejo del sistema lingüístico del género hacia los 3 años (Hernández-Pina, 1984; Soler, 1984). Es muy posible que la claridad, regularidad y productividad del sistema de género en español ayude a los niños a dominarlo, jugando la información intralingüística un papel facilitador muy importante. Los datos que poseemos sobre errores que cometen los niños en la marcación del género y la concordancia de género, indican que prestan gran atención a los aspectos formales del lenguaje. Errores de sobremarcación como *mota rota o "tierra azula cometidos por un niño sobre los 21-25 meses (Hernández-Pina, 1984; se pueden encontrar otros ejemplos semejantes en Clark, 1985), o errores de sobrerregulación de los sufijos de género de una lengua a otra en el caso de una niña bilingue catalán- 
castellano a los 32 meses ("conillo en vez de "conill", "conejo» en catalán, por interferencia del sufijo masculino /-o/ del castellano) (Vila, 1984), indican la sensibilidad de los niños a las claves intralingüísticas.

El papel facilitador de las claves intralingüísticas (sufijos, artículos...) se pone de manifiesto al comparar el proceso de adquisición en niños que están expuestos a sistemas lingüísticos distintos, unos con riqueza y variedad de formas de marcación del género, y otros con una pobreza mayor. Por ejemplo, Mills (1986) ha hallado que los niños ingleses adquieren los pronombres personales más tarde que los niños alemanes. Aquellos cometen significativamente más errores hacia los 3 años de edad que los niños alemanes, quienes ya poseen a esa edad una alta competencia en su uso correcto. Este resultado indica que - excluyendo otros factores- cuanto más extensivo y productivo es el sistema de marcación del género en una lengua, más fácil es su aprendizaje, puesto que proporciona una información más frecuente y concordante que puede ser utilizada por el niño. En la medida en que los pronombres personales de tercera persona son la única parte de la lengua inglesa con marcación gramatical de género, es obvio que los niños ingleses tendrán mucha menos práctica con el género que los niños alemanes, lengua que es mucho más rica en este respecto. Se puede decir que sus posibilidades de buscar regularidades en el sistema son mucho menores. De nuevo, estos datos apuntan a la importancia de las claves intralingüísticas en la adquisición del género.

Otros datos provenientes del alemán, adicionalmente, también apuntan a la gran importancia de la terminación fonológica y la sintaxis en la adquisición de diferentes partes del sistema de género (McWhinney, 1978; Schneuwly, 1978; Böhme, 1983; Mills, 1986), aun cuando Mills (1986) también ha mostrado el papel jugado por factores semánticos, como la frecuencia o el rasgo animado.

La transparencia en la marcación del género es un factor que debe tener su importancia en el proceso, tal como se puede apreciar al comparar lo que ocurre con los niños alemanes y los españoles. Aunque no se ha realizado ningún estudio comparativo específico, los datos aportados por Mills (1986) indican que los niños alemanes tienen más dificultades que los españoles (nuestros datos) en el dominio del sistema de género. Las diferencias entre los dos sistemas deben ser las causas de esta disparidad. El alemán, que utiliza declinaciones para marcar los diferentes casos, realiza la marcación del género y el del caso mediante un mismo sufijo que, por tanto, cubre varias funciones. A esta plurifuncionalidad del sufijo utilizado para marcar el género, hay que añadir la mayor variedad de formas, así como su menor regularidad. Esto hace del alemán una lengua en la que el aprendizaje del género no sea tan fácil como en español, que, en este aspecto, es muy regular, simple, y transparente.

Los resultados hallados por mí coinciden, en líneas generales, con los de Karmiloff-Smith (1979), obtenidos mediante el empleo de una técnica muy similar, aun cuando con menos posibles combinaciones de las claves en juego, con niños franceses. Estos utilizaban también las claves intralingüísticas (sufijo y artículo) con mucha mayor predilección que las extranlingüísticas (género natural), siendo su importancia muy semejante a la que tiene en sus coetáneos españoles. En todo caso, se observa una relevancia incluso algo menor del género natural en los niños españoles. Las propie- 
dades estructurales de ambas lenguas las hacen muy semejantes en este aspecto de la marcación del género, lo cual probablemente explica la coincidencia tan grande en los resultados de ambos estudios (vease Pérez Pereira, 1988, y no publicado, para una comparación más detallada).

La comparación translingüística del estadio en que los niños llegan a dominar los morfemas de marcación de género da sustento a la afirmación de Slobin (1982: 56) de que la adquisición de aquellos sistemas lingüísticos en que hay distinciones claras y binarias que ofrecen criterios formales para la adición de sufijos se realiza con más facilidad que la de aquellos sistemas que carecen de estas características. De hecho, los niños que aprenden sistemas con tres géneros, y con marcadores morfofonológicos ambiguos, escasamente transparentes y poco predecibles (como el alemán (Mills, 1986), checo (Henzl, 1975), servo-croata (Radulovic; 1975), o ruso (Slobin, 1966, Popova, 1973, Zakharova, 1973), parecen hacerlo más tarde que los niños que deben aprender lenguas con dos géneros, y con una marcación del género claramente diferenciado y sistemático (como el árabe hablado en Egipto (Omar, 1970), hebreo (Levi, 1983, Berman, 1985), francés (KarmiloffSmith, 1979), o español (Hernández-Pina, 1984, y los datos del presente trabajo). Incluso comparando dos lenguas estructuralmente muy semejantes, como el ruso y el polaco, se aprecia que ciertas diferencias que hacen de esta última un sistema más consistente y claro, son las responsables de que los niños polacos adquieran con menos dificultades que los rusos el sistema de marcación del género (Smoczinska, 1985: 646). Este hecho lleva a la autora a realizar una afirmación que es absolutamente coincidente con la posición adoptada en este trabajo: que los niños son «capaces de aprender reglas que están basadas en criterios totalmente arbitrarios, con tal de que estos criterios sean consistentes y suficientemente claros para ser descubiertos" (Smoczinska, 1985: 646).

\section{CONCLUSIONES}

De los resultados obtenidos en el experimento es posible derivar algunas conclusiones:

1) Los niños son capaces de dominar la marcación del género de las palabras y la concordancia de género entre los elementos de la oración antes de los 4 años de edad. Al no existir diferencias importantes entre los sujetos de 4 y 11 años de edad, se puede decir que el sistema de género del español está asimilado hacia esa edad.

2) En esa tarea de aprendizaje los niños descansan fundamentalmente en la información que proporciona el propio lenguaje (claves morfofonológicas y sintácticas), siendo la información extralingüística escasamente relevante. La importancia de las claves sintácticas parece incrementarse ligeramente a las edades estudiadas.

3) La teoría del género natural no puede explicar los resultados obtenidos, que apoyan la concepción según la cual los niños trabajan también sobre las propiedades formales del lenguaje en sus esfuerzos por dominar el sistema lingüístico.

Comparando los resultados obtenidos con los sujetos hispanoparlantes con los de otras investigaciones realizadas sobre el tema con los niños ha- 
blantes de otras lenguas, es posible adelantar algunas conclusiones generales tentativas sobre las características del proceso de adquisición del género.

1) Los niños tienen la capacidad de prestar atención y a sacar provecho de la existencia de claves intralingüísticas en la determinación del género de los nombres y de la concordancia de género.

2) Las claves intralingüísticas o formales, cuando existen en una lengua, parecen ser incluso más importantes que las claves extralingüísticas ya desde una edad muy temprana, como revelan los estudios longitudinales, con tal de que dichas claves sean lo suficientemente claras.

3) Cuantas más claves coincidentes proporcione un lenguaje, más fácilmente se adquirirá el sistema de género. $\mathrm{O}$, para decirlo con otras palabras, la existencia de claves intralingüísticas en un determinado idioma facilita el aprendizaje de la marcación del género en algunas partes del habla tales como, por ejemplo, los pronombres (la comparación de las producciones correctas de pronombres entre niños ingleses y alemanes es crucial al respecto).

4) Cuanto más regulares, transparentes y productivos sean los medios lingüísticos para marcar el género, más rápidamente se adquirirá el sistema lingüístico del género en una lengua determinada. La no plurifuncionalidad de los marcadores de género ayuda a los niños en la adquisición del sistema, tal como el hebreo, español o francés, por un lado, y el ruso, álemán o servo-croata, por el otro. Por tanto la adquisición se facilita cuando existe una correspondencia entre una sola forma con una sola función o significado (transparencia y claridad), tal como ha propuesto Slobin (1985). La regularidad es también un factor importante, como ha quedado claro en la comparación entre la adquisición del género en ruso y en polaco.

Estos puntos centrales e interconectados entre sí de las conclusiones deben considerarse a la luz de las características de cada sistema lingüístico y de las partes del habla bajo consideración, como Mills (1986) ha indicado. Pero esa necesidad de concreción no niega el hecho absolutamente importante de que los niños están dotados con la capacidad de tener en cuenta la información proporcionada por el sistema lingüístico del género propiamente.

Estas conclusiones parecen afianzar aquellas posiciones teóricas sobre la adquisición del lenguaje (Karmiloff-Smith, 1983, 1986; Bowerman, 1985; Levy, 1988; Pérez Pereira 1988) que defienden que los niños también prestan atención y trabajan sobre la información lingüística «per se» en sus esfuerzos por dominar el lenguaje.

De igual modo, y como una implicación de lo anterior, se oponen a la visión de la primacía de la experiencia con la realidad extralingüística como fuente del desarrollo, y hablan en favor de una concepción de las relaciones entre lenguaje y pensamiento que, superando las concepciones del determinismo cognitivo. El ámbito de la experiencia lingüística también es importante para el desarrollo (y no sólo lingüístico) del niño. 


\section{Referencias}

ANDERSON, J. M. (1961). The morphophonemics of gender, in Spanish nouns. Lingua. X, 285-296.

Bates, E.; Benigni, L.; Bretherton, I.; Camaioni, L.; y Volterra, V. (1977). From gesture to the first word: on cognitive and social prerequisites. En $M$. Lewis y $L$. A. Rosemblum (Ed.) Interaction, conversation, and the development of languaje. Nueva York: John Wiley \& Sons.

Bem, S. L. (1981). Gender Schema Theory: A cognitive account of sex typing. Psychological Review. 84, 354-364.

Berman, R. A. (1985). The acquisition of Hebrew. En D. I. Slobin (Ed.), The crosslinguistic study of language acquisition. Volume 1: The data. Hillsdale: Lawrence Erlbaum Associates.

BÔHME, K. (1983). Children's understanding and awareness of German possesive pronouns. Doctoral Dissertation. University of Nijmegen, Holland.

BOWERMAN, M. (1985). Beyond communicative adequacy: From piecemeal knowledge to an integrated system in the child's acquisition of language. En K. E. Nelso (Ed.), Children's language. Volume 5. Hilsdale: Lawrence Erlbaum.

Cl.ARK, E. V. (1985). The acquisition of Romance, with special reference to French. En D. I. Slobin (Ed.), The crosslinguistic study of language acquisition. Volume 1: The data. Hillsdale: $\mathrm{N} \mathrm{J}$. Lawrence Erlabaum Associates.

DEUTSCH, W. y PECHMAN, T. (1978). Ihr, dir, or mir? On the acquisition of pronouns in German children. Cognition. 6, 155-168.

FAGOT, B. I.; LEINBACH, M. D. y HAGAN, R. (1986) Gender labeling and the adoption of sextyped behaviors. Developmental Psychology. 22, 440-443.

GarCia MADRUGA, J. (1988). Lenguaje y conducta cognitiva: el papel del lenguaje en la solución de problemas. Estudios de Psicologia, 33/34, 191-210.

GATHERCOLE, V.C. (1985), "He has too much hard questions": the acquisition of the linguistic mass-count distinction in much and many. Journal of Child Language. 12, 395-415.

Gelman, S. A.; Collman, P. y MACCOBY, E. E. (1986). Inferring properties from categories versus inferring categories from properties: The case of gender. Child Develpoment. 57, 396-404.

GoRDON, P. (1985). Evaluating the semantic categories hypothesis: The case of the count/mass distinction. Cognition. 20, 209-242.

GreenberG, J. H. (1966). Language universals. En T. A. Sebeok (Ed.) Current trends in Linguistics. Vol. 3. The Hague: Mouton.

Henzl, V. M. (1975). Acquisition of grammatical gender in Czech. Papers and Reports on Child Language Development. 10, 188-198.

HERNÁNDEZ-PINA, F. (1984). Teorias psicociolingüisticas y su aplicación a la adquisición del español como lengua materna. Madrid: Siglo XXI.

IвRAHIM, M. H. (1973). Grammatical gender. The Hague: Mouton.

KaIL, M. y Charvillat, A. (1988). Local and topological processing in sentence comprehension by French and Spanish children. Journal of Child Language. 15, 537-662.

KaRmiLoff-SMITH, A. (1979). A functional approach to child language. A study of determiners and reference. Cambridge; Cambridge University Press.

KARMILOFF-SMITH, A. (1983). Language development as a problem-solving process. Papers and Reports on Child Language Development. 22, 1-22.

KARMILOFF-SmITH, A. (1986). Some recent issues in the study of language acquisition. En J. Lyons, R. Coates, M. Deuchar, y G. Gazdar (Eds.) New horizons in Linguistics 2. Harmondsworth: Penguin Books.

KATZ, N.; BAKER, E. y MACNAMARA, J. (1974). What's in a name? A study of how children learn common and proper names. Child Development. 65, 469-473.

LEVY, Y. (1983a). The acquisition of Hebrew plurals: The case of missing gender category. Journal of Child Language. 10, 107-121.

LEVY, Y. (1983b). It's frogs all the way down. Cognition. 15, 75-93.

LEVY, Y. (1988). On the early learning of formal grammatical systems: evidence from studies of the acquisition of gender and countability. Journal of Child Language. 15, 179-187.

LÓPEZ, F. (1984). Adquisición de rol y de la identidad sexual. Infancia y Aprendizaje, 26, 25-40.

LOPEZ, F. (1988). Adquisición y desarrollo de la identidad sexual y de género. En J. Fernández (Ed.), Nuevas perspectivas en el desarrollo del sexo y el género. Madrid: Pirámide.

Macnamara, J. (1972). Cognitive basis fo language learning in infants. Psychological Review, $79,1,1-13$.

MACWHINNEY, B. (1978). The acquisition of morphophonology. Monographs of the Society for Research in Child Development. Serial 43, No. 1-2.

MARATSOS, M. (1983). Some current issues in the study of the acquisition of grammar. En P. H. Mussen (Ed.) Handbook of child Psychology. Vol. 3. Nueva York. Wiley.

MARATSOS, M. (1988). The acquisition of formal word classes. En Y. Levy, I. Schlesinger, y 
M. Braine (Eds.) Categories and processes in language acquisition. Hillsdale: Lawrence Erlbaum.

Maratsos, M. P. y Chalkely, M. A. (1980). The internal language of children syntax: The ontogenesis and representation of syntactic categories. En K. E. Nelson (Ed.) children's language. Vol. 2. Nueva York. Gadner Press.

MARTiNEZ, J. A. (1977). Los elementos de la gramática y el género en castellano. En VV.AA. Estudios ofrecidos a Emilio Alarcos Llorach. Tomo I. Oviedo.

MiLLs, A. (1986). The acquisition of gender. A study of English and German. Berlin. SpringerVerlag.

MULFORD, R. (1983). Semantic and formal factors in the comprehension of Icelandic pronoun gender. Papers and Reports on Child Language Development. 22, 83-91.

MULFORD, R. (1985). Comprehension of Icelandic pronoun gender: Semantic versus formal factors. Journal of Child Language. 12, 443-453.

OMAR, M. E. (1970). The acquisition of Egyptian Arabic as a native language. Doctoral Dissertation. Georgetown University.

Pérez Pereira, M. (1984). Lenguaje y pensamiento en el desarrollo: Hacia un nuevo modelo teórico. Estudios de Psicologia, 17, 117-130.

PÉREZ-PEREIRA, M. (1988). La atención a factores intralingüisticos en la adquisición del lenguaje. Estudios de Psicología. 34/35, 211-227.

PÉREZ-PEREIRA, M. (1988). The acquisition of gender by Spanish children: Some comparisons with English and other languages. Comunicación presentada en el Annual Meeting of the British Psychological Society. Developmental Section. Harlech, 16-19 septiembre.

PÉREZ-PEREIRA M. (no publicado). Semantic versus formal theories of gender acquisiton: A criticism of Mulford's study. Enviado para su publicación al Journal of Child Language.

PÉREZ-PEREIRA, M. (no publicado). The acquisition of gender: What Spanish children tell us? Enviado para su publicación al Journal of Child Language.

Popova, M. I. (1973). Grammatical elements of language in the speech of pre-school children. En C. A. Ferguson and D. I. Slobin (Eds.). Studies of Child Language Development. Nueva York: Holt, Rinehart and Winston.

Radulovic, L. (1975). Acquisition of language: Studies of Dubrovnik children. Tesis Doctoral. University of California, Berkeley.

ROSEMBLAT, A. (1962). Morfología del género en español. Comportamiento de las terminaciones en -o, -a Nueva Revista de Filología Hispánica. XVI, 31-80.

SCHLESINGER, I. M. (1981). Semantic assimilation in the development of relational categories. En W. Deutsch (Ed.) The child's construction of language. Londres: Academic Press.

SCHNEUWLY, B. (1978). Zum Erwerb des Genus im Deutschen: Eine Mögliche Strategie. Manuscript, Max-Plank-Institut, Nijmegen, Holland.

SECO, R. (1973). Manual de gramática española. Madrid: Aguilar.

SERBIN, I. A. y SPRAFKIN, C. (1986). The salience of gender and the process of sex typping in three-to seven-year-old children. Child Development. 57., 1188-1199.

SLOBIN, D. I. (1966). The acquisition of Russian as a native language. En F. Smith and G. A. Miller (Eds.), The genesis of language. Cambridge (Mass.): M. I. T. Press.

SLOBIN, D. I. (1973). Cognitive prerequisites for the development of grammar. En C. A. Ferguson y D. I. Slobin (Eds.) Studies of Child Language Development. Nueva York: Holt, Rinehart and Winston.

SLOBIN, D. I. (1982). La construcción de la gramática por el niño. En M. Monfort (Comp.) Los trastornos de la comunicación en el niño. I Simposium de Logopedia. Madrid: CEPE.

SLOBIN, D. I. (1985). Crosslinguistic evidence for the language-making capacity. En D. I. Slobin (Ed.), The crosslinguistic study of language acquisition. Volume 2: Theoretical issues. Hilsdale: Lawrence Erlbaum.

SMOCZYNSKA, M. (1985). The acquisition of Polish. En D. I. Slobin (Ed.), The crossilinguistic study of language acquisition. Volume 1: The data. Hillsdale: $\mathrm{N}$ J. Lawrence Erlbaum Associates.

SOLER, R. (1984). Adquisición y utilización del artículo. En M. Siguán (Ed.), Estudios sobre psicología del lenguaje infantil. Madrid: Pirámide.

TUCKER, G. R.; LAMBERT, W. E. y RIGAULT, A. (1977). The French speacker's skill with grammatical gender: an example of rule-governed behavior. The Hague: Mouton.

VILA, I. (1984). Yo siempre hablo catalán y castellano: Datos de una investigación en curso sobre la adquisición del lenguaje en niños bilingües. En M. Siguán (Coord.) Adquisición precoz de una segunda lengua. Barcelona: Publicacións i Edicións de la Universitat de Barcelona.

ZaKharova, A. V. (1973). Acquisition of forms of grammatical case by preschool children. En C. A. Ferguson y D. I. Slobin (Eds.), Studies of Child Language Development. Nueva York: Holt, Rinehart and Winston. 


\section{Extended summary}

Regarding the acquisition and development of gender two opposing theoretical positions have been defended. According to the first, children rely on extralinguistic clues (semantic or natural gender) to learn linguistic marking of gender (Mulford, 1985). Contrary to this, other authors support that children pay attention primarily to intralinguistic information (suffix, determiners, etc.). Both theories are closely related to two views on language acquisition which may be called "semantic based theory" (Slobin, 1973), and «formal learning theory» (Maratsos and Chalkley, 1980).

The aim of the present research study was to test whether Spanish speaking children rely on either intralinguistic or extralinguistic clues when learning the linguistic gender system.

In Spanish there are three possible informational clues that children can use to determine the gender of a noun and the agreement of other variable elements acompanying it. These clues are 1) natural gender or semantic information provided by the sexual features of some living beings; 2) gender suffix of the nouns which are considered feminine whenever they end in /-a/, or masculine if nouns end in /-o/ (Rosemblat, 1962; Anderson, 1961); and 3) syntactic information furnished by preceeding determiners, such as articles or demostratives, which have different forms according to their gender.

An experimental manipulation of these clues was undertaken to produce a series of items offering the range of possible combinations. These were 1) items with only one of the clues present; 2 ) items with a combined effect of two clues in agreement (i.e. both of them feminine or masculine); and 3) items where clues were in conflict (e.g., one of them masculine and the other feminine). This experimental manipulation enabled us to test the relative strength of the different types of competing clues. Artificial nouns were presented to children within a particular frame. Subjects were asked to recognize the gender of these nouns and to establish the agreement of other variable elements acompanying it. A procedure similar to that used by Karmiloff-Smith (1979) was employed in the experiment.

Subjects were 160 Spanish speaking children from 4 to 11 years of age, distributed in 8 age groups with 20 subjects in each group.

The results obtained show that Spanish children pay far more attention to syntactic and morphophonological information than to semantic information. Thus, our results with Spanish children support the formal theory of gender acquisition and not the natural gender theory. Comparisons with other studies carried out with children who speak other languages indicate that the more regular, transparent and productive the linguistic gender marking devices are, the faster children master the linguistic system of gender. 